\title{
Relevance of Predictors in Difficult Intubation for Patients Undergoing Maxillofacial Surgery
}

\author{
Shalender Sharma1 ${ }^{*}$, Kaberi Majumder ${ }^{2}$, Krishna Kishor ${ }^{1}$, Ripan Das $^{1}$, Shiv Kishor ${ }^{3}$, \\ Sunil Gulia ${ }^{1}$ \\ ${ }^{1}$ Department of Oral and Maxillofacial Surgery, SGT Dental College, Gurgaon, India \\ ${ }^{2}$ Department of Orthodontics, SGT Dental College, Gurgaon, India \\ ${ }^{3}$ Department of General Surgery, SPS Apollo Hospitals, Ludhiana, India \\ Email: "sharma.shalender@rediffmail.com
}

Received 3 November 2014; revised 2 December 2014; accepted 18 December 2014

Copyright (C) 2014 by authors and Scientific Research Publishing Inc.

This work is licensed under the Creative Commons Attribution International License (CC BY).

http://creativecommons.org/licenses/by/4.0/

(c) (i) Open Access

\begin{abstract}
Objective: To determine a clinically useful variable for predicting difficult tracheal intubation in patients with seemingly normal airways. Material and Methods: In our study we had included 68 patients from September 2011 to September 2013 who needed tracheal intubation for elective maxillofacial surgery. An airway assessment test was conducted on each patient prior to general anaesthesia, with respect to mouth opening, sternomental distance, thyromental distance, oropharyngeal (Mallampati) classification and ability to protrude the mandible. After induction of anaesthesia, the laryngeal view during laryngoscopy was graded and then the ability to intubate was assessed. Results: Incidence of difficult intubation occurred in $8(11.76 \%)$ cases out of 68 patients. Airway test that was significant for predicting difficult tracheal intubation was SMD of less than $12.5 \mathrm{~cm}$, TMD of less than $6 \mathrm{~cm}$, a score according to Mallampati $e t$ al. of greater than III, protrusion of mandible position $B$ and position $C$ and IIG less than $3 \mathrm{~cm}$ with sensitivity of $87.5 \%$, $62.5 \%, 62.5 \%, 25 \%$ and $50 \%$, respectively. Conclusion: Our study concluded that sternomental distance had the highest sensitivity of $87.5 \%$ which was statistically significant $(p<0.05)$ compared to other parameters.
\end{abstract}

\section{Keywords}

Difficult Intubation, Sternomental Distance, Airway, Mallampatti Score, Oral Surgery

\footnotetext{
${ }^{*}$ Corresponding author.
}

How to cite this paper: Sharma, S., Majumder, K., Kishor, K., Das, R., Kishor, S. and Gulia, S. (2014) Relevance of Predictors in Difficult Intubation for Patients Undergoing Maxillofacial Surgery. International Journal of Clinical Medicine, 5, $1365-1373$. http://dx.doi.org/10.4236/ijcm.2014.521174 


\section{Introduction}

The failure to maintain a patient's airway following the induction of general anaesthesia is a major concern not only for anaesthesiologist but also for the operating surgeon. As an oral \& maxillofacial surgeon we often have to operate on difficult airway cases in the head \& neck region under general anaesthesia. For securing the airway tracheal intubation using direct laryngoscopy remains the method of choice in most cases. However direct laryngoscopic intubation is difficult in $1.2 \%$ of cases and impossible in very few of cases, who have seemingly normal airways [1].

The unanticipated difficult laryngoscopic intubation places patients at increased risk of complications ranging from sore throat to mortality. Maintaining a patient's airway is essential for adequate oxygenation \& ventilation; failure to do so even for a brief period of time can be life threatening. Approximately 600 patients die each year in a developed world from complications related to airway management, and the scenario in the under developed world is much grimmer [2]. Unexpected death is probably the result of lack of accurate predictive test for difficult intubation and inadequate preoperative examination.

The American Society of Anaesthesiologist (ASA) defines the difficult airway as "the clinical situation in which a conventionally trained anaesthesiologist experiences difficulty with mask ventilation, difficulty with tracheal intubation, or both". The difficult endotracheal intubation is defined as "proper insertion of tracheal tube with conventional laryngoscopy requiring more than three attempts or more than ten minutes" (ASA, 2000) [3].

As difficult intubation occurs infrequently and is not easy to define, research has been directed at predicting difficult laryngoscopy, i.e., when it is not possible to visualize any portion of the vocal cords after multiple attempts at conventional laryngoscopy. It is argued that if difficult laryngoscopy has been predicted and intubation is essential, skilled assistance and specialist equipment should be provided. Although the incidence of difficult or failed tracheal intubation is comparatively low, unexpected difficulties and poorly managed situations may result in a life threatening condition or even death [4].

Difficulty in intubation is usually associated with difficulty in exposing the glottis by direct laryngoscopy. This involves a series of manoeuvres, including extending the head, opening the mouth, displacing and compressing the tongue into the submandibular space and lifting the mandible forward. The ease or difficulty in performing each of these manoeuvres can be assessed by one or more parameters [5].

An accurate prediction of difficulty in intubation might reduce the frequency of additional maneuvers (i.e. awake intubation). Patients with difficult intubation can be identified by careful examination of anatomical landmarks and certain clinical factors. However, it is still questioned whether true prediction is possible and which variables should be chosen for evaluation [6].

Several preoperative airway assessment tests have been used singularly or in various combinations but they are all characterized by low sensitivity, reasonable specificity and low positive predictive values. This is because difficult intubation is uncommon, but this does not mean that one should not predict difficult intubation utilizing some of the useful bedside assessment methods. This has a benefit of alerting both the surgeon and anaesthesiologist to consider the problems in intubation and plan accordingly to achieve an unobstructed airway [4].

In this study our goals were to evaluate the incidence of difficult intubation and to calculate the specificity and sensitivity of the measured parameters and most important, to find the best combination in order to predict difficult intubation.

\section{Material and Methods}

This study was conducted on 68 patients who were scheduled to undergo oral and maxillofacial surgery under general anaesthesia from September 2011 to September 2013.

Informed consent was taken from all the patients of either sex who were included in the study. Edentulous patients were excluded to avoid introduction of a variable that may independently affect the predictability of difficult intubation. Patients requiring rapid sequence intubation, with history of difficult intubation, unstable cervical spine and anatomical abnormality of head and neck were excluded from study.

The airway was assessed pre-operatively in the pre-induction room on the day of surgery by the same anaesthetist who was involved in the anaesthetic care of the patient. The data of the patient was entered on a proforma. The information collected included patients' age, sex, weight, height, dentition (normal or buck teeth) and airway test that could be easily completed at bed side. 
These airway tests were:

\subsection{Sternomental Distance (SMD)}

Patients were asked to assume the sniffing the morning air position by tilting the head up as far as possible with mouth closed (the investigators hand is placed on the lower neck to assume immobility of the lower cervical spine). The straight distance between the upper border of the manubrium sterni and the symphysis mentae was measured. Distance was approximated to nearest $0.5 \mathrm{~cm}$ (Figure 1).

\subsection{Thyromental Distance (TMD)}

Patients were asked to assume the sniffing morning air position. The straight distance between the thyroid notch $\&$ the symphysis mentae was measured. Distance was approximated to nearest $0.5 \mathrm{~cm}$ (Figure 2).

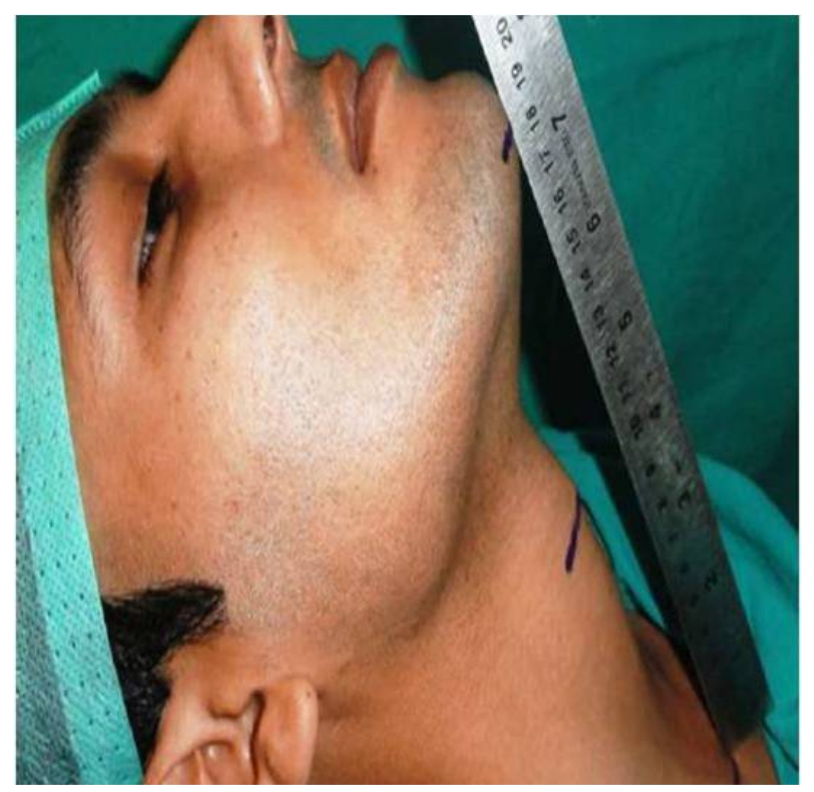

Figure 1. Measurement of sternomental distance.



Figure 2. Measurement of thyromental distance. 


\subsection{Mallampati Score}

The patients were made to sit erect with mouth opened maximally; tongue protruded maximally, while the observation was done from patient eye level an inspection was done of the pharyngeal structure with the help of a pen torch without the patient phonating. The view is then graded as (Figure 3).

Class 1. Faucial pillar, soft palate \& uvula could be visualized.

Class 2. Faucial pillar, soft palate could be visualized but uvula was masked by base of the tongue.

Class 3. Only soft palate could be visualized.

Class 4. Soft palate could not be visualized (Samson \& Young's modification).

\subsection{Protrusion of Mandible}

The patients were asked to protrude the chin as far as forward as possible protrusion was graded (Figure 4).

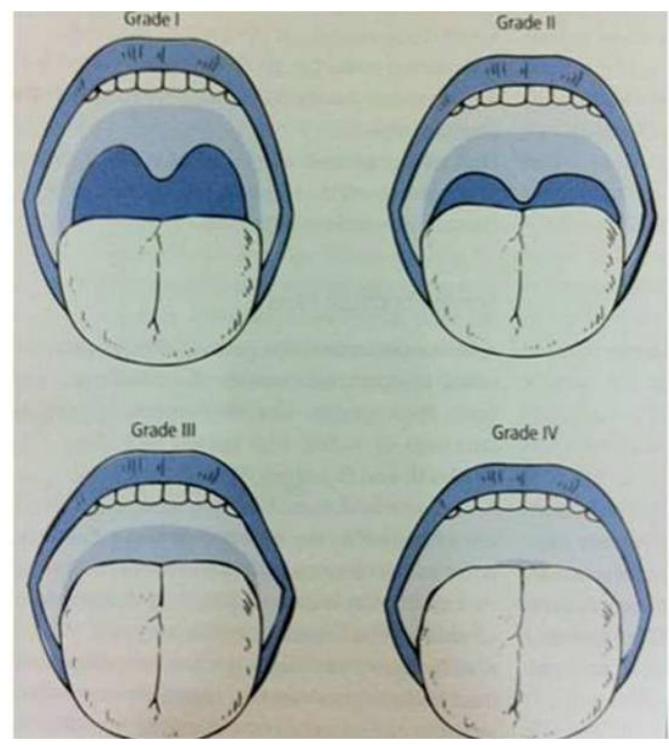

Figure 3. Malllampatti classification.

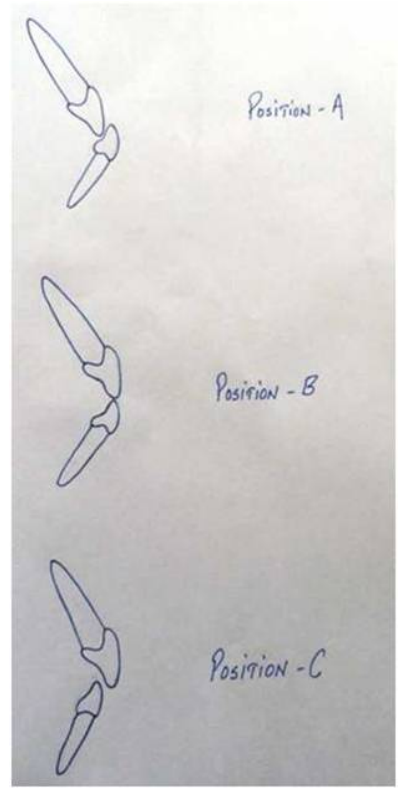

Figure 4. Mandibular protrusion position. 
Position A: If the lower incisors could be protruded anterior to the upper incisors.

Position B: If the upper \& lower incisors could touch.

Position C: If the lower incisors could not be brought forward to touch the upper incisors.

\subsection{Inter Incial Gap}

The patients were made to sit erect and were asked to open the mouth as far as possible and the distance between the upper \& lower incisor teeth were measured.

Each test from 1 to 5 was repeated thrice to rule out any error.

\subsection{Method of Anaesthesia}

Patients were induced with injection thiopentone sodium $5 \mathrm{mg} / \mathrm{kg}$, Nalbuphine $0.1 \mathrm{mg} / \mathrm{kg}$ and Atracurium 0.5 $\mathrm{mg} / \mathrm{kg}$, and larngoscopy was performed after three minutes. Points noted during intubation included size of blade needed, whether tracheal pressure was applied, the best view of larngoscopy and the number of attempts.

The definition of difficult larngoscopic intubation was based on the best laryngoscopic view and number of larngoscopy attempts since it has been shown that using both these parameter improves the reliability of identification of difficult laryngoscopic tracheal intubation.

The view at larngoscopy was graded by Cormack Lehane in the following manner:

Grade I: If part of vocal cord visible.

Grade II: If only the arytenoids were visible.

Grade III: If only epiglottis was visible.

Grade IV: If epiglottis was not visible.

Difficult intubation in our study was defined as number of larngoscopy attempts + grade of larngoscopy, the score $<4$ or 4 was taken as easy intubation and a score $>4$ as difficult intubation.

\subsection{Statistical Analysis}

Patient data assessed using the chi-square test where appropriate. Statistical significance was assumed when $p<$ 0.05 . Each test was evaluated by its sensitivity, specificity and positive predictive value (PPV).

\section{Results}

A total number of 68 patients with ages ranging from 20 - 60 years were studied. Amongst them 40 were males and 28 were females. All these patients were required general anaesthesia for oral and maxillofacial surgical procedures.

Patient's characteristics like sex, sternomental distance, thyromental distance, mallampatti score, mandible protrusion, inter incisor gap and their relation to the Cormack Lehane grades shown in Table 1. Airway test that were significant for predicting difficult tracheal intubation was SMD of less than $12.5 \mathrm{~cm}$, TMD of less than 6 $\mathrm{cm}$, a score according to Mallampati et al. of greater than III, protrusion of mandible position B and position C and IIG less than $3 \mathrm{~cm}$.

The incidence of difficult intubation assessed by five variables was 8 patients (11.76\%) out of 68 patients. Amongst this one case was Cormack lehane grade II which was intubated on third attempt, five cases were of grade III-four were intubated on third attempt and one was intubated by manipulation, and one case was of grade IV which was intubated with the help of laryngeal mask (Table 2).

In our studies we have obtained the following values of sensitivity and positive predictivity respectively (Table 3).

1) Sternomental distance: $87.5 \%$ and $31.8 \%$.

2) Thyromental distance: $62.5 \%$ and $15.6 \%$.

3) Mallampati: $62.5 \%$ and $10.2 \%$.

4) Protrusion of mandible: $25 \%$ and $11.8 \%$.

5) Interincisal gap: $50 \%$ and $8.2 \%$.

Sternomental distance had the highest sensitivity of $87.5 \%$ and PPV of $31.8 \%$ which was statistically significant $(p<0.05)$ compared to other parameters. 
Table 1. Assesment between laryngoscopic view and patient’s variables.

\begin{tabular}{|c|c|c|c|c|c|}
\hline Variables & No. of cases & CL I \& II & CL III \& IV & Easy & Difficult \\
\hline Sex male/female & $40 / 28$ & $32 / 22$ & $8 / 6$ & $35 / 23$ & $5 / 3$ \\
\hline \multicolumn{6}{|l|}{ Sternomental distance } \\
\hline$<12.5 \mathrm{~cm}$ & 10 & 7 & 3 & 2 & 6 \\
\hline$>12.5 \mathrm{~cm}$ & 58 & 57 & 1 & 56 & 2 \\
\hline \multicolumn{6}{|l|}{ Thyromental distance } \\
\hline$<6.5 \mathrm{~cm}$ & 18 & 16 & 2 & 15 & 13 \\
\hline$>6.5 \mathrm{~cm}$ & 50 & 47 & 3 & 48 & 2 \\
\hline \multicolumn{6}{|l|}{ Mallampati score } \\
\hline 1 & 24 & 23 & 1 & 24 & 0 \\
\hline 2 & 17 & 17 & 0 & 15 & 2 \\
\hline 3 & 16 & 13 & 3 & 14 & 2 \\
\hline 4 & 11 & 9 & 2 & 10 & 1 \\
\hline \multicolumn{6}{|l|}{ Mandible position } \\
\hline A & 53 & 52 & 1 & 52 & 1 \\
\hline B & 15 & 14 & 1 & 14 & 1 \\
\hline \multicolumn{6}{|l|}{ Inter incisor gap } \\
\hline$<3.5 \mathrm{~cm}$ & 12 & 11 & 1 & 9 & 3 \\
\hline$>3.5 \mathrm{~cm}$ & 56 & 54 & 2 & 55 & 1 \\
\hline
\end{tabular}

Table 2. Difficult intubations according to laryngoscopic view.

\begin{tabular}{|ccc|}
\hline Cormack & Intubation & Intubation \\
\hline Lehane grades & Difficult & Easy \\
\hline I & 52 & - \\
II & 8 & 1 \\
III & & 5 \\
IV & & 2 \\
\hline
\end{tabular}

Table 3. Sensitivity, specificity and positive predictive value of various tests.

\begin{tabular}{ccccccc}
\hline Test & $\begin{array}{c}\text { Predicted difficult } \\
\text { intubation }\end{array}$ & $\begin{array}{c}\text { Difficult case } \\
\text { detected }\end{array}$ & $\begin{array}{c}\text { Sensitity } \\
(\%)\end{array}$ & $\begin{array}{c}\text { Specificity } \\
(\%)\end{array}$ & $\begin{array}{c}\text { Positive } \\
\text { predictive value }\end{array}$ & $\begin{array}{c}\text { Significance } \\
p<0.05\end{array}$ \\
\hline $\begin{array}{c}\text { Sternomental } \\
\text { distance (cm) }\end{array}$ & 22 & 8 & 87.5 & 81.7 & 31.8 & 0.00001 \\
$\begin{array}{c}\text { Thyromental } \\
\text { distance (cm) }\end{array}$ & 32 & 5 & 62.5 & 67.1 & 15.6 & 0.09533 \\
Mallampatti score & 49 & 5 & 62.5 & 46.3 & 10.2 & 0.63173 \\
$\begin{array}{c}\text { Protrusion of } \\
\text { mandible (A-C) }\end{array}$ & 17 & 2 & 25 & 81.7 & 11.8 & 0.64363 \\
Interincisal gap & 49 & 4 & 50 & 45.1 & 8.2 & 0.79144 \\
\hline
\end{tabular}

\section{Discussion}

One of the major challenges in the practice of general anesthesia is to predict how difficult the intubation of patients will be, in the preoperative period. Pregnant patients, those suffering from facial/maxillary trauma, those 
with small mandibles or intra-oral pathology such as infections or tumours are all more likely to present difficulties during intubation. Patients who suffer with rheumatoid disease of the neck or degenerative spinal diseases often have reduced neck mobility making intubation harder. In addition spinal cord injury may result from excessive neck movements during intubation attempts. Poor teeth and the inability to open the mouth are obvious other factors as are obesity, and inexperience on the part of the anaesthetist [7].

King T. A., Adams A. P. et al. documented that more than 600 patients die annually as a result of failed intubation [8]. The relationship between a problematic airway, difficult laryngoscopy and difficult intubation has not been documented properly in the literature.

In our study, the difficult intubation rate was determined to be $11.25 \%$. Here, we preferred to use repetitive attempts as the criterion for difficult intubation, using the definition assigned by the ASA. Wilson M. E. reported that the incidence of difficult intubation in the operating room was reported to range from $1.5 \%$ to $8.5 \%$ [2]. In our study, the incidence of difficult intubations was relatively higher compared with that in previous studies. We believe this occurred because our group of patients consisted of patients with maxillofacial anomalies including retrognathia, micrognathia, facial asymmetry, and TMJ ankylosis, in which mouth opening was limited.

Anaesthetists at times encounter difficult intubation in an apparent normal individual in spite of using the best positioning and profound relaxation [6]. When recognized before attempts at tracheal intubation, virtually all difficult airways can be secured by the selected use of specialized tracheal intubation techniques, like intubating LMA, LMA CTrach [9], fibreoptic laryngoscope and senascope [10], although many of these methods require special training, experience, assistance and equipments.

S. Rao Mallampati, Stephen P. et al. conducted a prospective study to develop a clinical sign to predict difficult tracheal intubation [3]. They suggested that the size of the base of the tongue is an important factor determining the degree of difficulty of direct laryngoscopy. They proposed a correlation between the appearance of oropharyngeal structures and laryngeal appearance by which difficult intubation cases can be identified. They have graded the view from class 1 to class 4 and same is the order of prediction of difficult intubation. This has been considered as one of the landmark articles in the history of preoperative airway assessment. In our study also the patients who were having grade 3 and grade 4 they were relatively difficult to intubate. The results from this test are influenced by the ability to open the mouth, the size and mobility of the tongue and other intra-oral structures and movement at the craniocervical junction.

M. E. Wilson, D. Spiegelhalter et al. identified five useful risk factors as predictors of difficult intubations [2]. According to them inter incisal gap less than $5 \mathrm{~cm}$ is a good predictor of difficult intubation. On the contrary to this study in our study the patients who were having interincisal gap less than $3 \mathrm{~cm}$ were at increased risk of difficult intubation.

C. M. Frerk conducted a study on 244 patients preoperatively with a modified Mallampati test and a measurement of thyromental distance [11]. According to him the patients who were having thyromental distance less than $7 \mathrm{~cm}$ were at increased risk of difficult intubation. On the contrary to this study in our study thyromental distance of less than $6.5 \mathrm{~cm}$ is a good predictor of difficult intubation.

Keyvan Karkouti, D. Keith Rose et al. had used mandibular protrusion as one of the three parameters for their model of predictors of difficult intubation [5]. In our study also position B and C of mandible was a good predictor of difficult intubation.

D. Savva assessed 352 consecutive patients before operation using the modified Mallampati test and by measuring thyromental \& sternomental distances, forward protrusion of the mandible and interincisor gap with mouth fully opened [12]. According to his study a sternomental distance of $12.5 \mathrm{~cm}$ or less is a good predictor of difficult intubation. In our study also the patients who were having sternomental distance less than $12.5 \mathrm{~cm}$ were relatively difficult to intubate.

S. A. Ramdhani, L. A. Mohammed et al. observed that the sternomental distance is a good predictor for difficult intubation in obstetric anaesthesia it had a sensitivity of 66.7\% [13]. In our study the sensitivity of sternomental distance for prediction of difficult intubation is $87.5 \%$.

D. A. Rocke, W. B. Murray, C. C. Rout et al. studied relative risk analysis of factors associated with difficult intubation in obstetric anaesthesia [14]. Potential risk documented were obesity, short neck, missing, protruding, or single maxillary incisors, receding mandible which is comparable with our study findings.

An ideal predictive test of difficult intubation should be able to distinguish all subjects with potentially difficult intubations from all those with easy intubations. This would mean a test with $100 \%$ sensitivity, specificity, PPV. 
Sunanda Gupta et al. reported that no single airway test can provide a high index of sensitivity and specificity for prediction of difficult airway [15]. Therefore it has to be a combination of multiple tests. It must be recognized, however, that some patients with a difficult airway will remain undetected despite the most careful preoperative airway evaluation. To avoid this error in our study we had taken multiple variables for the prediction of difficult intubation.

Naguib M., Scarnrnan et al. suggested that the sensitivity of a prediction model is more important than its specificity, and should be weighted more heavily when determining the model to be used [16]. C. K. Koay proved that assessment of multiple anatomical features would improve prediction of difficult intubation [17]. Assessment of receding chin, neck extension, mouth opening, teeth, tongue size, thyromental distance might pick up $81 \%$ of difficult airways.

Aysegul Mine Tuzuner-Oncul had suggested that the grading systems of Mallampati et al. and Cormack and Lehane, "when used alone, had the highest sensitivity values 59\% and 75\%, respectively" [18]. The highest PPV was determined in patients with an IIG of less than 2 (100\%). our study showed minimal positive predictive value with interincisal gap and statistically insignificant sensitivity was exhibited by Mallampati Grade ( $p=$ 0.631 ) in predicting difficult intubation. However sternomental distance had the heights sensitivity of $87.5 \%$ and PPV of $31.8 \%$ which was statistically significant $(p<0.05)$ compared to other parameters.

In clinical practice there are several factors that may contribute to the lower sensitivity estimates, for example, if patients do not follow instructions appropriately or consistently or find it difficult to assess a position, reliability estimates will be lowered. To increase the reliability of the tests, patients need to have the required maneuvers clearly described and when necessary even to have them demonstrated; asking the patients to repeat the maneuvers until performed correctly will also help [19].

Although the definition of difficult intubation is related to the concept of limited laryngoscopic view, we find that laryngeal grade-III is not completely suitable to characterize difficult intubation as in some patients in our study with CL grade-III, intubation was done by simple manipulation without using an aid.

\section{Conclusion}

In conclusion difficult intubation is a multifactorial problem. Our study showed that there was no single, simple test to predict difficulty in tracheal intubation and sternomental distance had the highest sensitivity of $87.5 \%$ which was statistically significant $(p<0.05)$ compared to other parameters. The combination of predictive tests can be used for improving sensitivity and PPVS.

\section{Conflict of Interest}

None identified.

\section{Financial Relationship with Organization}

We don’t have any financial relationship for our research.

\section{References}

[1] Cattano, D., Panicucci, E., Paolicchi, A., et al. (2004) Risk Factors Assessment of Difficult Airway: An Italian Survey of 1956 Patients. Anesthesia \& Analgesia, 99, 1774-1779. http://dx.doi.org/10.1213/01.ANE.0000136772.38754.01

[2] Wilson, M.E., Spiegelhalter, D. and Robertson, J. (1988) A Predicting Difficult Intubation. British Journal of Anaesthesia, 61, 211-216. http://dx.doi.org/10.1093/bja/61.2.211

[3] Mallampati, S.R., Gatt, S.P. and Desai, S.P. (1985) A Clinical Sign to Predict Difficult Intubation. Canadian Anaesthetists Society Journal, 32, 429-434. http://dx.doi.org/10.1007/BF03011357

[4] El-Gazouri, A.R., McCarthy, R.J., Tuna, K.J., et al. (1996) Preoperative Airway Assessment: Predictive Value of Multivariate Index. Anesthesia \& Analgesia, 82, 1197-1204.

[5] Karkouti, K., Rose, D.K., Ferris, L.E., et al. (1996) Interobsever Reliability of Ten Tests for Predicting Difficult Tracheal Intubation. Canadian Journal of Anesthesia, 43, 554-557. http://dx.doi.org/10.1007/BF03011765

[6] Arne, J., Descoins, P., Fusciardi, J., et al. (1998) Preoperative Assessment for Difficult Intubation in General and ENT Surgery: Prediction Value of a Clinical Multivariate Risk Index, Clinical Investigation. British Journal of Anaesthesia, 80, 140-146. http://dx.doi.org/10.1093/bja/80.2.140 
[7] American Society of Anesthesiologists (2013) Practice Guidelines for Management of the Difficult Airway: An Updated Report. Anesthesiology, 118, 251-270. http://dx.doi.org/10.1097/ALN.0b013e31827773b2

[8] King, T.A., Adams, A.P., et al. (1990) Failed Tracheal Intubation. British Journal of Anaesthesia, 65, 400-412. http://dx.doi.org/10.1093/bja/65.3.400

[9] Liu, E.H.C., Goy, R.W.L. and Chen, F.G. (2006) The LMA CTrach, a New Laryngeal Mask Airway for Endotracheal Intubation under Vision. British Journal of Anaesthesia, 96, 396. http://dx.doi.org/10.1093/bja/ael001

[10] Biro, P., Bättig, U., Henderson, J. and Seifert, B. (2006) First Clinical Experience of Tracheal Intubation with the Sensascope, a Novel Steerable Semirigid Video Stylet. British Journal of Anaesthesia, 97, 255-261. http://dx.doi.org/10.1093/bja/ael135

[11] Frerk, C.M. (1991) Predicting Difficult Intubation. Anaesthesia, 46, 1005-1008. http://dx.doi.org/10.1111/j.1365-2044.1991.tb09909.x

[12] Savva, D. (1994) Prediction of Difficult Tracheal Intubation. British Journal of Anaesthesia, 73, 149-153. http://dx.doi.org/10.1093/bja/73.2.149

[13] Ramdhani, S.A., Mohammed, L.A., Rocke, D.A., Gouws, E. and Ramadhani, S.A. (1996) Sternomenatal Distance as a Sole Predictor Difficult Intubation in Obstetrics Anesthesia. British Journal of Anaesthesia, 77, 312-316. http://dx.doi.org/10.1093/bja/77.3.312

[14] Rocke, D.A., Murray, W.B., Rout, C.C. and Gouws, E. (1992) Relative Risk Analysis of Factors Associated with Difficult Intubation in Obstetric Anesthesia. Anesthesiology, 77, 67-73. http://dx.doi.org/10.1097/00000542-199207000-00010

[15] Gupta, S., Sharma, R. and Jain, D. (2005) Airway Assessment: Predictors of Difficult Airway. Indian Journal of Anaesthesia, 49, 257-262.

[16] Naguib, M., Scarnrnan, F.L., O’Sullivan, C., Aker, J., Ross, A.F., Kosmach, S. and Ensor, J.E. (2006) Predictive Performance of Three Multivariate Difficult Tracheal Intubation Models: A Double-Blind, Case-Controlled Study. Anesthesia \& Analgesia, 102, 818-824.

[17] Koay, C.K. (1995) Difficult Tracheal Intubation-Analysis and Management in 37 Cases. Singapore Medical Journal, 39, 112-114.

[18] Tuzuner-Oncul, A.M. and Kucukyavuz, Z. (2008) Prevalence and Prediction of Difficult Intubation in Maxillofacial Surgery Patients. Journal of Oral and Maxillofacial Surgery, 66, 1652-1658.

[19] Tse, J.C., Rimm, E.B. and Hussian, A. (1995) Predicting Difficult Intubation in Surgical Patients Scheduled for General Anesthesia: A Prospective Blind Study. Anesthesia and Analgesia, 81, 254-258. 
Scientific Research Publishing (SCIRP) is one of the largest Open Access journal publishers. It is currently publishing more than 200 open access, online, peer-reviewed journals covering a wide range of academic disciplines. SCIRP serves the worldwide academic communities and contributes to the progress and application of science with its publication.

Other selected journals from SCIRP are listed as below. Submit your manuscript to us via either submit@scirp.org or Online Submission Portal.
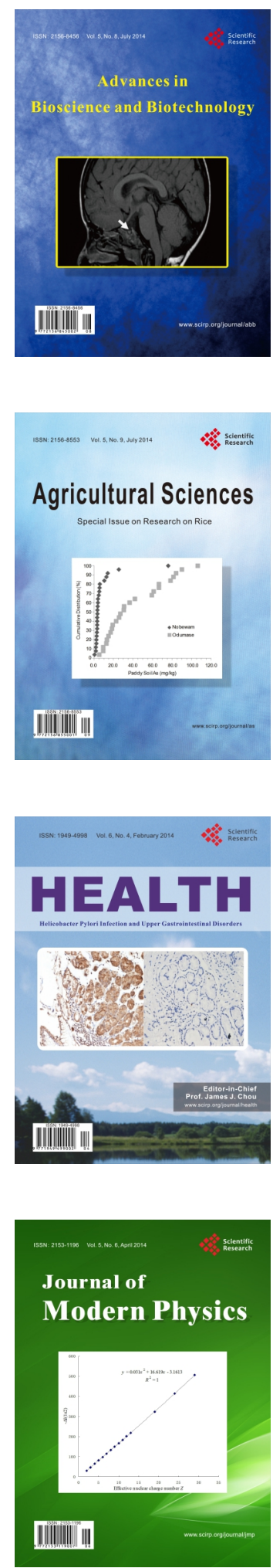
